\title{
The International Physical Activity Questionnaire modified for the elderly: aspects of validity and feasibility
}

\author{
Anita Hurtig-Wennlöfl,*, Maria Hagströmer ${ }^{2}$ and Lovisa A Olsson ${ }^{3}$ \\ 'Department of Clinical Medicine, School of Health and Medical Sciences, Örebro University, SE-701 82 \\ Örebro, Sweden: ${ }^{2}$ Department of Neurobiology, Care Sciences and Society, Division of Physiotherapy, \\ Karolinska Institutet, Stockholm, Sweden: ${ }^{3}$ Department of Laboratory Medicine, Örebro University Hospital, \\ Örebro, Sweden
}

Submitted 22 April 2009: Accepted 6 January 2010: First published online 3 March 2010

\begin{abstract}
Objective: To modify the self-administered, short version of the International Physical Activity Questionnaire (IPAQ) for adults to be used in the elderly (aged 65 years and above), and to validate this modified IPAQ for the elderly (IPAQ-E). Design: A direct validity study using accelerometer-measured physical activity (PA) as the criterion measure, and an indirect criterion validity study using highsensitivity C-reactive protein (hs-CRP) as a biological marker of activity.

Setting: Organisations for retired persons in Sweden.

Subjects: The direct validity study consisted of fifty-four participants and the indirect criterion validity study consisted of 359 participants. All participants were retired persons (66-91 years) living independently.

Results: All self-reported activity domains (sitting, walking, moderate and vigorous) were positively correlated with the corresponding variable objectively assessed by an accelerometer $(\rho=0 \cdot 277-0 \cdot 471)$, but a systematic error was observed. The specificity of IPAQ-E to identify low-active participants was $85 \%$, and the sensitivity to identify the more active participants was $81 \%$. A main effect of IPAQ-E category (Low, Moderate or High) was observed for hs-CRP $(P=0 \cdot 041)$.

Conclusions: We found this modified version of IPAQ, the IPAQ-E, to be well accepted by our sample of socially active elderly. It provided acceptable estimates of PA, well in line with other questionnaires, even though it had a systematic error. The IPAQ-E was able to identify an expected response of a biomarker (hsCRP) to PA. We recommend the use of the IPAQ-E to classify participants aged 65 years and above into PA categories, to rank individuals or to identify individuals meeting certain PA criteria.
\end{abstract}

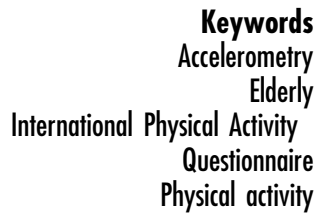

Keywords

Elderly

Questionnaire

Physical activity
To promote and maintain health, regular physical activity (PA) is essential. In both men and women, individuals with a high level of PA have been shown to have a longer life expectancy than more sedentary individuals ${ }^{(1,2)}$. More recently, positive effects of PA have also been reported on cognitive functions ${ }^{(3,4)}$ as well as on depression and anxiety disorders ${ }^{(5)}$. The level and pattern of PA and various health markers have been extensively studied in young and middle-aged groups, but not to the same extent in older adults ${ }^{(6)}$. The elderly (aged 65 years and above) are a rapidly growing age group in many countries, and therefore merit special attention with regard to PA behaviour and its effect on health.

Most PA assessment methods have been developed for young-to-middle-aged participants, and just a few activity questionnaires have been developed specifically for the elderly participants ${ }^{(7)}$. This holds true also for the International Physical Activity Questionnaire (IPAQ, available at www.ipaq.ki.se ${ }^{(8)}$ ), which was developed by an international consensus group in 1998, primarily for international surveillance studies of PA in adults aged 18-65 years $^{(9)}$. The short-format IPAQ ${ }^{(10)}$ and the long-format IPAQ $^{(11)}$ have been validated in Swedish adults, but the modification and use of self-administered IPAQ in the elderly has not been validated before ${ }^{(12)}$.

The aims of the present study were: (i) to report the cultural adaptation of IPAQ to elderly participants, aged 65 years and above, in Sweden; (ii) to assess the direct criterion validity of the modified IPAQ using accelerometry; and (iii) to assess the indirect criterion validity of the modified IPAQ by using an established biomarker of PA (C-reactive protein; CRP). 


\section{Methods}

\section{Cultural adaptation of the IPAQ}

The Swedish translation of the short-format IPAQ for selfadministration in adults covering the last $7 \mathrm{~d}^{(8)}$ formed the base for the modification work. Throughout the modification process of the questionnaire to suit older adults, the guidance on cultural adaptation ${ }^{(13)}$ was used and, accordingly, a pilot test was performed. A few participants who were taking part in a book club organised by a senior citizens' organisation ( $n$ 15; nine women, mean age $=71$ years) were interviewed as they completed each item, and their comments and questions were used in the development of the modified IPAQ.

\section{Direct criterion validity}

A measure of validity of the IPAQ modified for elderly participants, hereafter called IPAQ-E, was evaluated by comparing the self-reported PA as assessed by IPAQ-E with PA objectively assessed by an accelerometer as a direct criterion instrument.

The accelerometer, ActiGraph, model GT1 M (Manufacturing Technology Inc., Fort Walton Beach, FL, USA) was used for the objective assessment of PA. This uniaxial accelerometer records vertical accelerations as 'counts', and provides both the duration and intensity (as counts/ time unit (epoch)) of PA. The instrument has been used in various settings and has been shown to provide valid information on PA patterns ${ }^{(14-17)}$. Data were sampled in $15 \mathrm{~s}$ epochs and the accelerometer was worn on an elastic belt around the waist for seven consecutive days during all waking hours, except during water activities. Data reduction and interpretation of the output were based on previous methodological studies ${ }^{(15-20)}$. Data were reduced using the ActiGraph analysis software MAHUffe (available from http://www.mrc-epid.cam.ac.uk/Research/ $\mathrm{PA} /$ Downloads.html ${ }^{(21)}$ ). Continuous periods of zero values exceeding $20 \mathrm{~min}$ were regarded as 'accelerometer not worn' and were not included in the calculation of total registered time ${ }^{(20)}$. Ten hours of registration per $\mathrm{d}$ were required for a day to be considered as valid, and at least four valid days of registration were required to include the registration in the statistical analysis. Activity count cut-off points applied to assign accelerometer outcomes to PA categories were as follows: time spent sitting was defined as where counts/min were $<100^{(17)}$, moderate activity (mixed lifestyle activities) was defined as activity resulting in 760-2019 counts/min ${ }^{(16)}$, moderate activity (ambulatory activities) was defined as producing 2020-4944 counts/min ${ }^{(19)}$ and vigorous activity was defined as $>4944$ counts $/ \min ^{(15)}$. Mean activity level was defined as total counts divided by total registered time (counts/min).

The IPAQ recommendations on data cleaning and processing $^{(22)}$ were followed with one exception: when no information was given for a specific intensity level (e.g. vigorous PA), this was entered as null activity on that level and the remaining information was used, not excluded as suggested in the protocol. The categorical outcome from the IPAQ assigns the participants into three PA categories (Low, Moderate and High) based on the reported time in combination with a weighting factor for the different activities (i.e. a factor $3 \cdot 3$ for walking, $4 \cdot 0$ for moderate PA and 8.0 for vigorous PA). If the reported times for walking, moderate and vigorous activities exceeded $180 \mathrm{~min} / \mathrm{d}$, data were truncated to be equal to $180 \mathrm{~min} / \mathrm{d}$, in accordance with the truncation of data rules $^{(22)}$. Here, we report time (min) spent in the different activities, with the information from the questionnaire calculated to give an average value per $\mathrm{d}$. The continuous IPAQ measures to be validated against the corresponding measures from accelerometry are the self-reported time spent sitting $(\mathrm{min} / \mathrm{d})$, moderate activity $(\mathrm{min} / \mathrm{d})$, walking $(\mathrm{min} / \mathrm{d})$ and vigorous activity $(\mathrm{min} / \mathrm{d})$. In addition to the IPAQ protocol categories (Low, Moderate and High), the participants were also categorised into two groups depending on whether they met the current PA recommendation for older adults from the American College of Sports Medicine/American Heart Association ${ }^{(23)}$ or not (Meeting the PA guidelines and Not meeting the PA guidelines). Briefly, the guidelines recommend $30 \mathrm{~min}$ of moderate PA per d; to be assigned to the group Meeting the PA guidelines based on IPAQ-E result, the participants had to be assigned to the category Moderate or High as described above, and based on accelerometry, at least 30 min of total moderate activity had to be recorded on average over all days.

Seventy participants were recruited through local branches of organisations for retired persons in the Örebro area (in Central Sweden), i.e a convenience sample from the target group of active elderly. Oral information about the purpose of the study and the practical procedures were given at local meetings. All participants provided written informed consent. Written information, accelerometers and questionnaires were thereafter delivered and were sent back by prepaid post. The participants were instructed to wear the accelerometer on an elastic belt around the waist during all time spent awake, except during water activities, for seven consecutive days. After completion of the accelerometer registration, the participants filled in the IPAQ-E, thus covering the same period of time (i.e. the last $7 d$ ). Data collection was conducted over 10 weeks, mainly during the autumn.

Completed accelerometer registrations and questionnaires were obtained in fifty-four participants: thirtyone women (57\%; aged 66-85 years) and twenty-three men (aged 66-82 years). Incomplete accelerometer data were returned by fourteen participants $(20 \%$ of total sample), and were due to accelerometer registrations of less than $10 \mathrm{~h} / \mathrm{d}$, and two questionnaires (3\% of total sample) were invalid due to incomplete data. There was no difference in gender distribution or age between 
the fourteen participants with missing data and the participants with valid data.

\section{Indirect criterion validity}

High levels of PA have been associated with low circulating levels of biomarkers of inflammation, e.g. $\mathrm{CRP}^{(24,25)}$. High-sensitive CRP (hs-CRP) was therefore chosen as the indirect criterion measure to check whether the IPAQ-E could detect the expected inverse relation between hs-CRP and PA.

The IPAQ-E was applied in a study of elderly participants (aged 65 years or above) called 'Active Seniors' study', originally designed to serve as a control group for clinical studies and including blood sampling for future analyses. The participants were recruited from the retired persons' organisations, which implies that they are independent and socially active. The locations for the recruitment were selected through a multistage sampling procedure aiming to represent a broad range of socioeconomic levels and include rural as well as urban and suburban areas. From the sampling frame of active seniors, 439 volunteered and 389 participated (89\%). Of the 389 participants, 359 (92\%) had valid data for both hs-CRP and IPAQ-E. There was no difference in gender distribution, age, height or weight in the thirty participants with missing data (hs-CRP, $n$ 7; IPAQ-E, $n$ 23) compared to the participants with a complete data set.

Blood samples were taken, with the participants in a supine position by venepuncture using vacuum tubes. Serum was obtained after clotting for 30-60 min at room temperature and centrifuging for $10 \mathrm{~min}$ at $2000 \mathrm{~g}$. All samples were stored at $-80^{\circ} \mathrm{C}$. The hs-CRP was analysed using a latex-enhanced immunoturbidimetry method, CRP (Latex) HS (from Roche/Boehringer Mannheim, Germany) on a Hitachi 911 multianalyser (Roche, Mannheim, Germany). The ethical committee of Örebro City Council approved the study (no. 274/02).

\section{Statistics}

The distribution of all continuous variables was checked. As most of the PA variables deviated from the normal distribution (Shapiro-Wilk's test for normality $P<0 \cdot 05$ ), non-parametric statistical analyses were used in the direct criterion validation study. Descriptive data are given as median and 25th-75th percentiles. The Mann-Whitney test was used to compare the categorical groups, and Spearman's $\rho$ was used for correlation analysis between the PA variables derived from the IPAQ-E and the accelerometer data. Scatter plots based on the Bland-Altman technique were used to provide an illustration of systematic and random error ${ }^{(26)}$. As an indicator of the systematic bias across the range of measured time spans, Spearman's $\rho$ was calculated. Cohen's $\kappa$ was used to test the agreement between different PA groups from IPAQ-E and accelerometer data. Percentage of agreement was also calculated, defined as the number of observations with perfect agreement between the two methods used. The ability of IPAQ-E to identify participants meeting certain PA criteria was also tested: specificity (here, the ability of the IPAQ-E to identify participants Not meeting the PA guidelines) and sensitivity (here, the ability of IPAQ-E to identify participants Meeting the PA guidelines).

In the indirect criterion validation study, the plasma hs-CRP concentrations required transformation to achieve a normal distribution and logarithmically transformed hs-CRP values were used in the ANOVA to test for the main effects between PA factors and hs-CRP. In the tables and the figure, untransformed data are provided for clarity. The SPSS for Windows statistical software package version 17 (SPSS Inc., Chicago, IL, USA) and the software VassarStats $^{(27)}$ were used, and the level of significance was set at $\alpha=0 \cdot 05$.

\section{Results}

\section{Cultural adaptation of the IPAQ}

During the process of modifying the Swedish version of the IPAQ to be more relevant for the elderly, we first changed only the activity examples to more age-relevant activities, and consensus was reached among the participants in the pilot group on the activities chosen and the description of intensity levels. However, during the pilot testing, we received negative reactions from the participants regarding the order of the questions. Originally, the question order was from vigorous to light activities (i.e. vigorous, moderate, walking and sitting). As most of the elderly pilot participants rarely participated in vigorous activities and seldom in moderate activities, they felt uncomfortable to answer these questions first. Therefore, we adapted the questionnaire by reversing the question order (i.e. sitting, walking, moderate and vigorous) to meet the real-life situation more closely and to focus on the main activities performed by this age group (sitting and walking). The layout was adjusted to facilitate reading by using the font size fourteen. The IPAQ-E is available on request from any of the authors.

\section{Direct criterion validity}

Descriptive data of the variables are given in Table 1. Age and time spent in different intensity levels of PA, selfreported or assessed by an accelerometer, did not differ between genders $(P>0 \cdot 05)$. Consequently, data were combined in the subsequent analyses. Twenty-four of the thirty-one women (77\%) and thirteen of the twentythree men (56\%) reported no vigorous PA in IPAQ-E. Associations between variables of self-reported and objectively assessed PA are shown in Table 2. Selfreported time spent sitting, the different moderate activities (separate and in combination) and vigorous activity were all significantly and positively correlated with the corresponding variable objectively assessed by an 
Table 1 Distributions of gender, age and self-reported physical activity from the International Physical Activity Questionnaire modified for the elderly (IPAQ-E) and accelerometry-assessed physical activity ( $n$ 54)

\begin{tabular}{|c|c|c|c|c|}
\hline & \multicolumn{2}{|c|}{ Women (n 31) } & \multicolumn{2}{|c|}{ Men $(n$ 23) } \\
\hline & Median & 25th-75th percentile & Median & 25th-75th percentile \\
\hline Age (years) & 74 & $69-77$ & 71 & $68-76$ \\
\hline \multicolumn{5}{|l|}{ Self-reported from IPAQ-E } \\
\hline Sitting $(\mathrm{min} / \mathrm{d})$ & 300 & $240-420$ & 420 & $300-600$ \\
\hline Moderate activity $(\mathrm{min} / \mathrm{d})$ & 14 & $1-36$ & 23 & $7-70$ \\
\hline Walking $(\min / \mathrm{d})$ & 43 & $17-120$ & 64 & $14-129$ \\
\hline Walking plus moderate activity $(\mathrm{min} / \mathrm{d})$ & 86 & $32-146$ & 137 & $17-231$ \\
\hline Vigorous activity $(\mathrm{min} / \mathrm{d})$ & 0 & $0-0$ & 0 & $0-17$ \\
\hline \multicolumn{5}{|l|}{ Accelerometer outcomes } \\
\hline Sitting $^{*}(\min / \mathrm{d})$ & 454 & $399-511$ & 518 & $435-565$ \\
\hline Light activity+ (min/d) & 200 & $172-241$ & 204 & $180-239$ \\
\hline Moderate, mixed lifestyle activitiesł ( $\mathrm{min} / \mathrm{d})$ & 59 & 41-79 & 65 & $37-82$ \\
\hline Moderate, ambulatory activities $\S(\mathrm{min} / \mathrm{d})$ & 23 & $13-32$ & 27 & $10-44$ \\
\hline Total moderate activities $(\mathrm{min} / \mathrm{d})$ & 85 & $65-99$ & 91 & $67-124$ \\
\hline Vigorous activityll (min/d) & 0 & $0-2$ & 0 & $0-5$ \\
\hline Mean activity level (counts/min) & 317 & $269-391$ & 315 & 197-409 \\
\hline Mean registered time & 736 & $696-815$ & 784 & $744-844$ \\
\hline
\end{tabular}

${ }^{*}$ Accelerometer cut-off point for sitting: $<100$ counts $/ \mathrm{min}^{(18)}$.

†Accelerometer cut-off points for light physical activities: $100-759 \mathrm{counts} / \mathrm{min}$.

$\ddagger$ Accelerometer cut-off points for moderate physical activity corresponding to mixed lifestyle activities: $760-2019 \mathrm{counts} / \mathrm{min}^{(17)}$

$\S$ Accelerometer cut-off points for moderate physical activity corresponding to ambulatory activities: $2020-4944$ counts/min ${ }^{(20)}$

IIAccelerometer cut-off point for vigorous physical activity: $>4944$ counts/min ${ }^{(16)}$.

Table 2 Spearman's rank correlation coefficients $(\rho)$ between self-reported physical activity from the International Physical Activity Questionnaire modified for elderly (IPAQ-E) and accelerometer data ( $n$ 54)

\begin{tabular}{|c|c|c|c|c|c|c|}
\hline \multirow[b]{2}{*}{$\begin{array}{l}\text { Self-reported } \\
\text { activity }\end{array}$} & \multicolumn{6}{|c|}{ Accelerometer data } \\
\hline & $\begin{array}{c}\text { Sitting } \\
(<100 \text { counts } / \\
\text { min })\end{array}$ & $\begin{array}{c}\text { Moderate, mixed } \\
\text { activities } \\
\text { (760-2019 counts/ } \\
\text { min) }\end{array}$ & $\begin{array}{c}\text { Moderate, } \\
\text { ambulatory activities } \\
\text { (2020-4944 counts/ } \\
\text { min) }\end{array}$ & $\begin{array}{l}\text { Total moderate } \\
\text { activities ( } 760 \text { to } \\
4944 \text { counts } / \mathrm{min} \text { ) }\end{array}$ & $\begin{array}{l}\text { Vigorous activity } \\
\text { (>4944 counts/ } \\
\text { min) }\end{array}$ & $\begin{array}{l}\text { Mean activity } \\
\text { level (mean } \\
\text { counts/min) }\end{array}$ \\
\hline Sitting & $0.277^{*}$ & $-0 \cdot 351^{* *}$ & -0.081 & $-0.337^{*}$ & 0.067 & -0.174 \\
\hline Walking & -0.178 & $0.338^{\star}$ & $0 \cdot 326^{\star *}$ & $0.363^{\star *}$ & $0 \cdot 211$ & $0 \cdot 297^{*}$ \\
\hline $\begin{array}{l}\text { Moderate } \\
\text { activity }\end{array}$ & $-0 \cdot 162$ & $0 \cdot 471^{\star \star \star}$ & $0 \cdot 168$ & $0 \cdot 396^{\star \star}$ & $0 \cdot 184$ & $0 \cdot 270^{*}$ \\
\hline $\begin{array}{l}\text { Walking plus } \\
\text { moderate }\end{array}$ & -0.209 & $0 \cdot 415^{\star \star}$ & $0 \cdot 340^{*}$ & $0 \cdot 414^{\star *}$ & $0 \cdot 260$ & $0 \cdot 347^{\star \star}$ \\
\hline $\begin{array}{l}\text { Vigorous } \\
\text { activity }\end{array}$ & 0.049 & $0 \cdot 117$ & $0 \cdot 161$ & 0.090 & $0.369^{\star *}$ & $0 \cdot 103$ \\
\hline
\end{tabular}

${ }^{\star} P<0.05 ;{ }^{* *} P<0.01 ;{ }^{* \star *} P<0.001$.

accelerometer, $\rho$ ranging from $0 \cdot 277$ to $0 \cdot 471$. There were also significant and negative correlation coefficients between self-reported time spent sitting and accelerometerassessed time in moderate activities, $\rho$ ranging from -0.337 to $-0 \cdot 351$.

Scatter plots based on the Bland-Altman technique for time spent sitting and in moderate activity level are shown in Figs $1 \mathrm{a}$ and $\mathrm{b}$, respectively, with the $x$ axis showing the average $\mathrm{min} / \mathrm{d}$ reported from both instruments. Both figures show that the IPAQ-E indicates less time than the accelerometer at the low end of the $x$ axis, whereas it indicates more time than the accelerometer as the average $\mathrm{min} / \mathrm{d}$ increased. There was a significant correlation between the differences between the instruments ( $y$ axis) and the average $\mathrm{min} / \mathrm{d}$ ( $x$ axis), $\rho=0 \cdot 370(P=0 \cdot 005 ; 95 \%$ CI $0 \cdot 114,0 \cdot 580)$ and $\rho=0 \cdot 600(P<0 \cdot 001 ; 95 \%$ CI $0 \cdot 396$, $0 \cdot 747$ ) for sitting and moderate activity, respectively.
According to the accelerometry data, seven of the fiftyfour participants (13\%) were active for less than $30 \mathrm{~min} / \mathrm{d}$ on average, corresponding to the Low IPAQ category and were thus not meeting the amount of activity recommended in the current guidelines for older adults. The specificity of IPAQ-E to identify these low-active participants was $85 \%$, whereas the sensitivity to identify the high-active participants was $81 \%$ (Table 3 ). Cohen's $\kappa$ for testing agreement was moderate $(\kappa=0.448$, $P<0 \cdot 001 ; 95 \%$ CI $0 \cdot 18,0 \cdot 72)$. The percentage of observations with perfect agreement between the methods was $82 \%$.

\section{Indirect criterion validity}

Descriptive data of selected variables in the Active Seniors' study are given in Table 4 . The distribution of the IPAQ categories in the sample was as follows: Low $15 \%$, 
Moderate $32 \%$ and High 53\%. The majority (64\%) did not report any vigorous $\mathrm{PA}$, and no gender differences were observed. Median (25th-75th percentile) for hs-CRP were $1.44(0 \cdot 84-2 \cdot 56)$ and $1.78(0 \cdot 79-3 \cdot 13) \mathrm{mg} / 1$ for women and men, respectively. A two-way ANOVA with the factors such as gender and IPAQ category (with levels Low, Moderate and High) showed that there was no interaction effect between gender and IPAQ categories, no main effect of gender, but a main effect of IPAQ category $(P=0 \cdot 041, P$ for linear trend $=0 \cdot 011)$ on ln-transformed hs-CRP. Figure 2 shows the mean values of hs-CRP across the IPAQ categories.
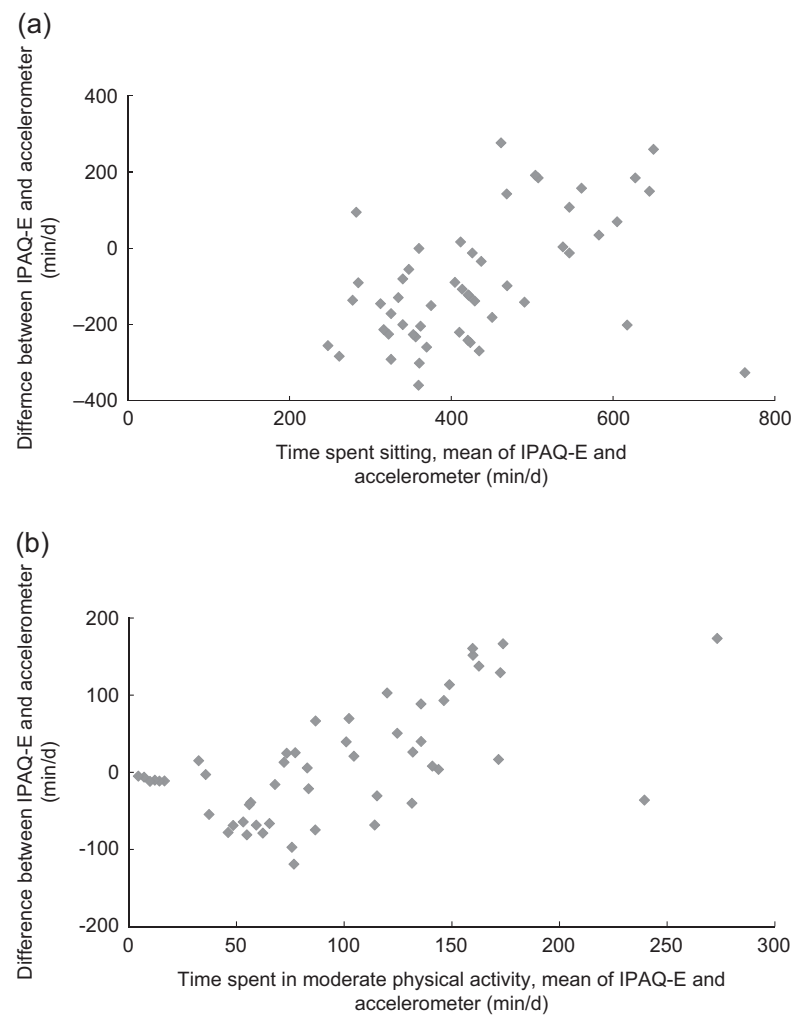

Fig. 1 (a) Scatter plot for time spent sitting as assessed by the International Physical Activity. Questionnaire modified for the elderly (IPAQ-E) and assessed by accelerometer. Spearman's $\rho=0.370(P=0.005 ; 95 \% \mathrm{Cl} 0 \cdot 114,0.580)$. (b) Scatter plot for time spent in moderate physical activity (walking and moderate physical activity) as assessed by IPAQ-E and accelerometer. Spearman's $\rho=0.600(P<0.001 ; 95 \% \mathrm{Cl}$ $0.396,0.747)$

\section{Discussion}

Here, we report different aspects of validity for the IPAQ$\mathrm{E}$, a culturally adapted version of IPAQ to be used in Swedish elderly persons (aged 65 years and above). After the cultural adaptations, the IPAQ-E was well accepted by the target population and the number of invalid questionnaires was low (6\%). The IPAQ-E was able to identify the expected relationship between PA and hs-CRP. The participants categorised into the Low PA group according to the IPAQ-E had a mean hs-CRP $>3 \mathrm{mg} / \mathrm{l}$. In clinical practice, hs-CRP $>3 \mathrm{mg} / \mathrm{l}$ is classified as indicating a high risk for a cardiac event ${ }^{(28,29)}$. This suggests that the IPAQ$\mathrm{E}$ was able to indicate participants having this risk factor.

In the comparison between self-reported PA assessed by IPAQ-E and objectively assessed PA by an accelerometer, the IPAQ-E showed similar properties as for other questionnaire versions of the IPAQ targeting adults at the age of 18-65 years. Correlation coefficients showed moderate correlations between IPAQ-E and the corresponding accelerometer variables $(\rho=0 \cdot 30-0 \cdot 40$, in general). The median correlation coefficient found in the international validation study preceding the launch of the IPAQ was $r=0 \cdot 30^{(9)}$. Two Swedish versions of IPAQ for adults have been validated separately. The long format was validated against the accelerometer (among other instruments) and showed the strongest correlations for vigorous PA $(\rho=0 \cdot 60-0 \cdot 70)$, while the comparison between self-reported sitting and the accelerometerassessed inactivity showed low correlation $(\rho=0 \cdot 17)^{(11)}$. The short format of IPAQ for adults has also been validated against the accelerometry and showed moderate correlation for total PA $(r=0 \cdot 34)$, while the sitting variables were weakly associated $(r=0 \cdot 16)^{(10)}$. The IPAQ-E showed a stronger association between self-reported sitting and sitting time as assessed by an accelerometer in the present study than the previously published validation studies in Swedish participants $(\rho=0 \cdot 277)$. Whether this result is attributable to the sequence in which the questions were presented has not been tested yet, but it could be a result of reporting sitting unbiased by (i.e. before) the other activities.

The impact of question order on self-reported PA has been studied by others ${ }^{(30-32)}$. In summary, Bolman et al. ${ }^{(30)}$ reported no influence on question order, while Hutto

Table 3 Number (\%) of participants classified as meeting the recommended physical activity (PA) level of at least 30 min of moderate activity per $d$ as assessed by the International Physical Activity Questionnaire modified for elderly (IPAQ-E) and assessed by an accelerometer $(n 54)$

\begin{tabular}{|c|c|c|c|}
\hline \multirow[b]{2}{*}{ Meeting PA guidelines as assessed by IPAQ-E } & \multicolumn{3}{|c|}{ Meeting PA guidelines as assessed by accelerometry } \\
\hline & No & Yes & Total \\
\hline No & $6(85)$ & 9 (19) & $15(28)$ \\
\hline Yes & 1 (15) & $38(81)$ & $39(72)$ \\
\hline Total & 7 (100) & $47(100)$ & $54(100)$ \\
\hline
\end{tabular}

Cohen's $\kappa$ for test of agreement, $\kappa=0.448 ; P<0.001 ; 95 \% \mathrm{Cl} 0 \cdot 18,0.72$. 
Table 4 Distributions of gender, age and self-reported physical activity as assessed by the International Physical Activity Questionnaire modified for the elderly (IPAQ-E), and high-sensitive C-reactive protein (hs-CRP) in the Active Senior study $(n$ 359)

\begin{tabular}{|c|c|c|c|c|}
\hline & \multicolumn{2}{|c|}{ Women ( $n$ 241) } & \multicolumn{2}{|c|}{ Men $(n 118)$} \\
\hline & Median & 25th-75th percentile & Median & 25th-75th percentile \\
\hline Age (years) & 74 & $69-79$ & 75 & $70-79$ \\
\hline \multicolumn{5}{|l|}{ Self-reported from IPAQ-E } \\
\hline Sitting $(\mathrm{min} / \mathrm{d})$ & 270 & $180-360$ & 270 & $225-360$ \\
\hline Moderate activity $(\mathrm{min} / \mathrm{d})$ & 75 & $10-180$ & 75 & $22 \cdot 5-150$ \\
\hline Walking (min/d) & 90 & $60-180$ & 60 & $45-120$ \\
\hline Walking plus moderate activity $(\mathrm{min} / \mathrm{d})$ & 210 & $120-300$ & 180 & $90-300$ \\
\hline Vigorous activity (min/d) & 0 & $0-60$ & 0 & 0-90 \\
\hline hs-CRP (mg/l) & $1 \cdot 44$ & $0.83-2 \cdot 51$ & $1 \cdot 75$ & $0 \cdot 75-2 \cdot 83$ \\
\hline
\end{tabular}

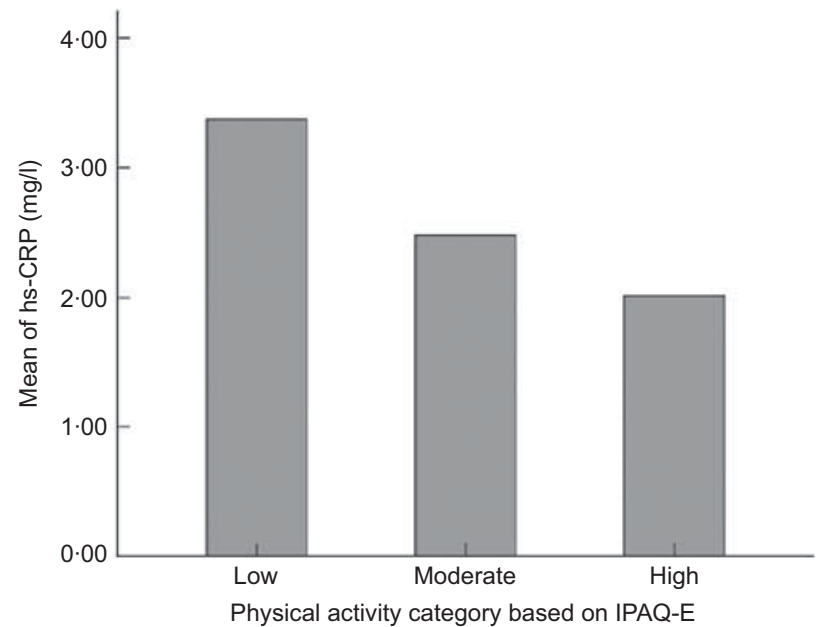

Fig. 2 Mean values of high-sensitivity C-reactive protein (hs-CRP) across physical activity groups as assessed by the International Physical Activity Questionnaire modified for the elderly (IPAQ-E). ANOVA for differences in In-transformed hs-CRP between IPAQ-E categories, $P=0.041, P$ for linear trend $=0.011$. The graph presents untransformed values

et $a .^{(31)}$ did find some influence of question order and recommended to present items concerning moderate and vigorous activities first, while items about walking seemed to be more robust and not susceptible to changes in question order. Similarly, Barnett et al. ${ }^{(32)}$ observed differences in vigorous activities across five independent samples using different question orders in the IPAQ, while no or minor differences were observed for walking or moderate PA or the proportion of participants meeting certain guidelines. Walking is generally the main contributor of PA in adults ${ }^{(33)}$, and walking as the PA of choice has been shown to increase with age ${ }^{(34)}$. In the elderly, vigorous PA is rare and does not contribute substantially to the total PA. The possible role of question order in the propensity to report PA might therefore have even less influence in the elderly. On the basis of comparisons with previously reported IPAQ validation studies, we instead suggest slightly improved reporting of sitting time by putting the sitting item first in the questionnaire. Taking part in a study makes you more observant of your PA behaviour and probably leads to higher correlation coefficients than would otherwise be the case. However, this is difficult to overcome and is a weakness in study design that we share with other validation studies.

The data analysis of output from objective instruments (e.g. 'counts' from an accelerometer) must also consider the specific study group, and there is a shortage of such studies in the elderly. Movement patterns and the energy cost of movement differ between age groups, leading to group-specific cut-off points to describe intensity levels and total amount of PA. Age-related changes in movement pattern including increased energy cost of walking $^{(35)}$ are assumed to be present among participants aged 65 years and above. The subjective experience of 'Moderate intensity' is therefore assumed to be reached at lower counts/min in the elderly than in younger adults. Different thresholds for activity counts corresponding to moderate (or other) intensity level exist. The cut-off points chosen in the present study are from different accelerometer studies ${ }^{(15,16,19)}$, and were chosen primarily based on the age distribution of the participants included in the study samples.

One of the few drawbacks with accelerometers is their incapability to accurately detect upper body movements when worn around the waist. Motion sensors in general are unable to distinguish between different types of walking conditions (e.g. flat, uphill or stairs or carrying loads). Furthermore, they cannot appropriately assess biking, rowing or resistance training. In Sweden, biking is quite a common method of transportation, even among the elderly, and missed accelerometer information from that specific activity could contribute to the observed discrepancies in IPAQ-E than accelerometer data (Fig. 1b). The error pattern is of similar magnitude as found in the IPAQ validation studies carried out in Swedish adults $^{(10,11)}$ and South African older adults ${ }^{(36)}$, as are the wide CI. In the scatter plot (Fig. 1b), we also see that low values on the $x$ axis indicate more PA assessed by an accelerometer than by IPAQ-E (negative values on the $y$ axis). This also seems relevant as the accelerometer 
detects all activity regardless of duration, while only activities with duration of $10 \mathrm{~min}$ or more are reported in the IPAQ. A similar finding, i.e. the error between the IPAQ and accelerometry, which increased as $\mathrm{min} / \mathrm{d}$ in different intensity PA reported in the IPAQ increased, has been reported for the long format of the IPAQ ${ }^{(37)}$.

Only a few participants in our study sample were classified into the Low IPAQ category. The accelerometer data also indicated a relatively high PA level. As discussed above, this is dependent on which cut-off points have been applied in the analysis of the accelerometer data. The only variable not depending on a cut-off point is the Mean activity level (total registered counts/total registered time, counts/min). When comparing the mean activity level in the present study with other studies, this variable also supported the finding that our sample was truly from a highly active population. The median value for the Mean activity level was $>300$ counts/min in both women and men in the present validation study. In the Better Ageing Project ${ }^{(6)}$, Mean activity levels in European (British, French and Italian) women and men aged 70 years and above of 236 and 255 counts/min, respectively, were reported. In the US National Health and Nutritional Examination Survey, the age group of 70 years and above reported mean activity levels of 180 and 389 counts/min in women and men, respectively. The mean activity level is, however, affected by registration time, which in turn is dependent on how data cleaning has been processed. We excluded continuous zero values of $>20$ min. By that procedure, total registered time/d was decreased, whereas mean counts/min were accordingly increased, as only 'active' periods of time were included in the analysis. All processes involved in the handling of the raw data make comparisons of accelerometer results across studies difficult.

In the present study, $20 \%$ of the accelerometer registrations failed to meet the inclusion criteria and were not included in data analysis. The main cause of exclusion was a registration time of less than $10 \mathrm{~h} / \mathrm{d}$. In the Better Aging Project ${ }^{(6)}$, a similar figure of about $20 \%$ of accelerometer registrations was also lost for various reasons. Thus, it may be prudent to allow for about one-fifth of accelerometer registrations to be missing in future studies of elderly participants, and to consider that in sample size calculations. The IPAQ-E showed, from this perspective, better feasibility than accelerometry, with only $6 \%$ of participants' missing data.

For several reasons, we described PA using time in min spent in different intensities, rather than converting into energy expenditure. First, the weighting factors used $(3 \cdot 3$ for walking, $4 \cdot 0$ for moderate PA and $8 \cdot 0$ for vigorous PA) correspond to activity-specific metabolic equivalent turnover values (MET values) in adults ${ }^{(38)}$. These MET values are most probably not appropriate for the elderly, e.g. moderate intensity level in the elderly is suggested to be lower than the same intensity level in younger participants ${ }^{(39)}$. However, we assume that they can still reflect the proportions of PA intensities, and are therefore useful for ranking participants with regard to PA. More studies on PA energy expenditure in the elderly and age-specific activity cut-off points are needed before energy expenditure calculations are supported. Second, time is a common unit for both instruments. Third, time in min gives an immediate idea of the amount of PA described.

In conclusion, we found this modified version of IPAQ for the elderly, the IPAQ-E, to be well accepted by a group of socially active elderly. It provided acceptable estimates of PA in this elderly group, well in line with other questionnaires, even though it has a systematic error. The IPAQ-E was able to identify an expected relationship between PA and a biomarker (hs-CRP). We recommend the use of the IPAQ-E to classify participants aged 65 years and above into PA categories to rank individuals or to identify individuals meeting certain PA criteria.

\section{Acknowledgements}

Nyckelfonden at Örebro City Council is acknowledged for the support to the Active Seniors' study. The study on Active Seniors was partly funded by Nyckelfonden, Örebro University Hospital. The authors have no conflicts of interest. A.H.W. was responsible for the validation study, the statistical analysis and drafting of the manuscript. M.H. provided technical support and reviewed the manuscript. L.A.O. conducted the data collection and the blood analysis in the study of the Active Seniors and reviewed the manuscript. All participants are gratefully acknowledged.

\section{References}

1. Paffenbarger RS Jr, Hyde RT, Wing AL et al. (1993) The association of changes in physical-activity level and other lifestyle characteristics with mortality among men. $N$ Engl J Med 328, 538-545.

2. Rockhill B, Willett WC, Manson JE et al. (2001) Physical activity and mortality: a prospective study among women. Am J Public Health 91, 578-583.

3. Angevaren M, Aufdemkampe G, Verhaar HJ et al. (2008) Physical activity and enhanced fitness to improve cognitive function in older people without known cognitive impairment. Cochrane Database Syst Rev issue 3, CD005381.

4. Lautenschlager NT, Cox KL, Flicker L et al. (2008) Effect of physical activity on cognitive function in older adults at risk for Alzheimer disease: a randomized trial. JAMA 300, 1027-1037.

5. Ströhle A (2009) Physical activity, exercise, depression and anxiety disorders. J Neural Transm 116, 777-784.

6. Davis MG \& Fox KR (2007) Physical activity patterns assessed by accelerometry in older people. Eur J Appl Physiol 100, 581-589.

7. Washburn RA (2000) Assessment of physical activity in older adults. Res Q Exerc Sport 71, S79-S88.

8. International Physical Activity Questionnaire (2009) International Physical Activity Questionnaire downloads. http:// www.ipaq.ki.se/downloads.htm (accessed 24 October 2009). 
9. Craig CL, Marshall AL, Sjostrom M et al. (2003) International Physical Activity Questionnaire: 12-country reliability and validity. Med Sci Sports Exerc 35, 1381-1395.

10. Ekelund U, Sepp H, Brage S et al. (2006) Criterion-related validity of the last 7-day, short form of the International Physical Activity Questionnaire in Swedish adults. Public Health Nutr 9, 258-265.

11. Hagstromer M, Oja P \& Sjostrom M (2006) The International Physical Activity Questionnaire (IPAQ): a study of concurrent and construct validity. Public Health Nutr 9, $755-762$.

12. Prince SA, Adamo KB, Hamel ME et al. (2009) A comparison of direct versus self-report measures for assessing physical activity in adults: a systematic review. Int J Behav Nutr Phys Act 5, 56.

13. International Physical Activity Questionnaire (2009) International Physical Activity Questionnaire - cultural adaptation. http://www.ipaq.ki.se/cultural.htm (accessed 24 October 2009).

14. Freedson PS, Melanson E \& Sirard J (1998) Calibration of the Computer Science and Applications, Inc. accelerometer. Med Sci Sports Exerc 30, 777-781.

15. Swartz AM, Strath SJ, Bassett DR Jr et al. (2000) Estimation of energy expenditure using CSA accelerometers at hip and wrist sites. Med Sci Sports Exerc 32, S450-S456.

16. Matthews CE (2005) Calibration of accelerometer output for adults. Med Sci Sports Exerc 37, S512-SS22.

17. Matthews CE, Chen KY, Freedson PS et al. (2008) Amount of time spent in sedentary behaviors in the United States, 2003-2004. Am J Epidemiol 167, 875-881.

18. Ward DS, Evenson KR, Vaughn A et al. (2005) Accelerometer use in physical activity: best practices and research recommendations. Med Sci Sports Exerc 37, S582-S588.

19. Troiano RP, Berrigan D, Dodd KW et al. (2008) Physical activity in the United States measured by accelerometer. Med Sci Sports Exerc 40, 181-188.

20. Alhassan S, Sirard JR, Spencer TR et al. (2008) Estimating physical activity from incomplete accelerometer data in field studies. J Phys Act Health 5, Suppl. 1, S112-S125.

21. Medical Research Council Epidemiology Unit (2009) MTI accelerometer analysis software (MAHUffe). http:// www.mrc-epid.cam.ac.uk/Research/PA/Downloads.html (accessed 24 October 2009).

22. International Physical Activity Questionnaire (2009) Guidelines for data processing and analysis of the International Physical Activity Questionnaire (IPAQ) - short and long forms, November 2005. http://www.ipaq.ki.se/scoring.pdf (accessed 24 October 2009).

23. Nelson ME, Rejeski WJ, Blair SN et al. (2007) Physical activity and public health in older adults: recommendation from the American College of Sports Medicine and the American Heart Association. Circulation 116, 1094-1105.
24. Kasapis C \& Thompson PD (2005) The effects of physical activity on serum C-reactive protein and inflammatory markers: a systematic review. J Am Coll Cardiol 45, $1563-1569$.

25. Plaisance EP \& Grandjean PW (2006) Physical activity and high-sensitivity C-reactive protein. Sports Med 36, 443-458.

26. Bland JM \& Altman DG (1986) Statistical methods for assessing agreement between two methods of clinical measurement. Lancet 1, 307-310.

27. Vassar College (2009) VassarStats. The confidence interval of rho. http://faculty.vassar.edu/lowry/rho.html (accessed 22 October 2009).

28. Buckley DI, Fu R, Freeman M et al. (2009) C-reactive protein as a risk factor for coronary heart disease: a systematic review and meta-analyses for the US Preventive Services Task Force. Ann Intern Med 151, 483-495.

29. Pearson TA, Bazzarre TL, Daniels SR et al. (2003) American Heart Association guide for improving cardiovascular health at the community level: a statement for public health practitioners, healthcare providers, and health policy makers from the American Heart Association Expert Panel on Population and Prevention Science. Circulation 107, 645-651.

30. Bolman C, Lechner L \& van Dijke M (2007) Question order in the assessment of misperception of physical activity. Int $J$ Behav Nutr Phys Act 4, 42-53.

31. Hutto B, Sharpe PA, Granner ML et al. (2008) The effect of question order on reporting physical activity and walking behavior. J Phys Act Health 5, Suppl. 1, S16-S29.

32. Barnett J, Nigg C, De Bourdeaudhuij I et al. (2007) The effect of item order on the physical activity estimates using the IPAQ. Californian J Health Promot 5, 23-29.

33. Simpson ME, Serdula M, Galuska DA et al. (2003) Walking trends among US adults: the Behavioral Risk Factor Surveillance System, 1987-2000. Am J Prev Med 25, 95-100.

34. Eyler AA, Brownson RC, Bacak SJ et al. (2003) The epidemiology of walking for physical activity in the United States. Med Sci Sports Exerc 35, 1529-1536.

35. Davies MJ \& Dalsky GP (1997) Economy of mobility in older adults. J Orthop Sports Phys Ther 26, 69-72.

36. Kolbe-Alexander TL, Lambert EV, Harkins JB et al. (2006) Comparison of two methods of measuring physical activity in South African older adults. J Aging Phys Act 14, 98-114.

37. Hagstromer M, Ainsworth BE, Oja P et al. (2010) Comparison of a subjective and an objective measure of physical activity in a population sample. J Phys Act Health (In the press).

38. Ainsworth BE, Haskell WL, Whitt MC et al. (2000) Compendium of physical activities: an update of activity codes and MET intensities. Med Sci Sports Exerc 32, S498-S504.

39. Tipton CE (2006) ACSM's Advanced Exercise Physiology. Baltimore, MD: Lippincott Williams \& Wilkins. 